This item was submitted to Loughborough's Research Repository by the author.

Items in Figshare are protected by copyright, with all rights reserved, unless otherwise indicated.

\title{
Seasonal performance of a-Si single- and multijunction modules in two locations
}

PLEASE CITE THE PUBLISHED VERSION

http://dx.doi.org/10.1109/PVSC.2005.1488423

PUBLISHER

(C) IEEE

VERSION

VoR (Version of Record)

LICENCE

CC BY-NC-ND 4.0

REPOSITORY RECORD

Gottschalg, Ralph, J.A. del Cueto, Thomas R. Betts, and David Infield. 2019. "Seasonal Performance of A-si Single- and Multijunction Modules in Two Locations”. figshare. https://hdl.handle.net/2134/8192. 
This item was submitted to Loughborough's Institutional Repository (https://dspace.lboro.ac.uk/) by the author and is made available under the following Creative Commons Licence conditions.

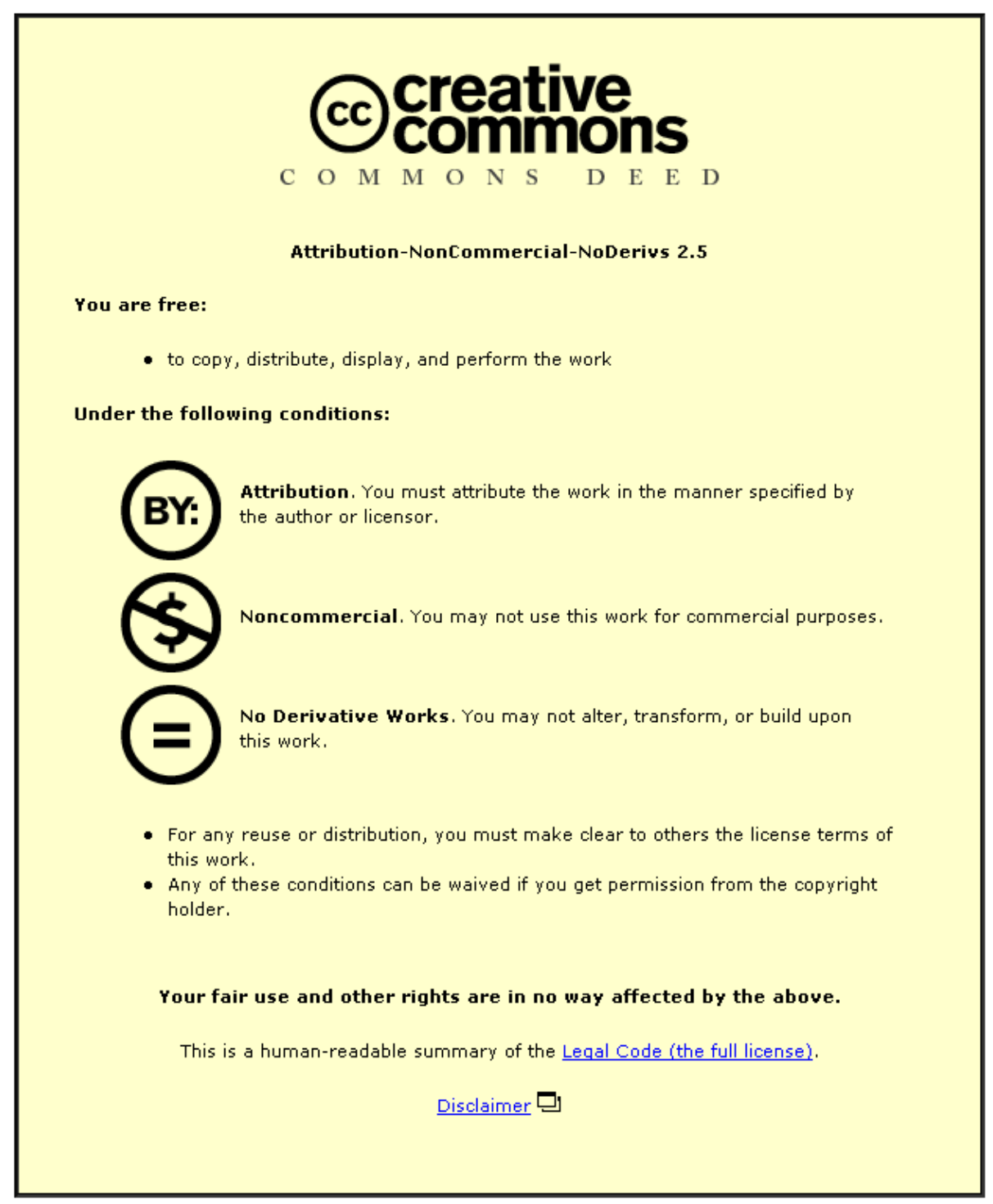

For the full text of this licence, please go to: http://creativecommons.org/licenses/by-nc-nd/2.5/ 


\title{
SEASONAL PERFORMANCE OF A-SI SINGLE- AND MULTIJUNCTION MODULES IN TWO LOCATIONS
}

\author{
R. Gottschalg', J.A. del Cueto ${ }^{2}$, T.R. Betts ${ }^{1}$, D.G. Infield ${ }^{1}$ \\ 1: CREST, Department of Electronic and Electrical Engineering, Loughborough University, Loughborough, LE11 \\ 3TU, UK \\ 2. NREL, National Renewable Energy Laboratory, 1617 Cole Blvd., Golden, CO 80401, USA
}

\begin{abstract}
Module performance data collected at two sites are analyzed in order to identify the respective magnitudes of seasonal annealing and degradation in comparison to spectral effects. It is demonstrated in this paper that at one site (Loughborough, UK) the spectrum dominates and very little seasonal annealing is observed. In contrast, at the other site (Golden, US), half of the seasonal variation can be attributed to spectral changes while the other half must be attributed to thermal annealing of defects. Differences beween multi-junction categories are investigated and it is shown that singlejunction devices exhibit a greater seasonal annealing than multi-junctions, while the latter tend to be more influenced by spectral effects.
\end{abstract}

\section{INTRODUCTION}

Amorphous silicon (a-Si) modules exhibit unusual performance variations in the course of the year typically, maximum operating efficiency is achieved in summer, when average module temperatures are highest. This results in higher performance ratios $(P R)^{1}$ in summer rather than in winter, which is highly advantageous for the annual energy production from these devices, especially considering that peak electrical demand in many countries occurs in the summertime. Several possible reasons for this behavior have been proposed, most significantly a shift in the incident
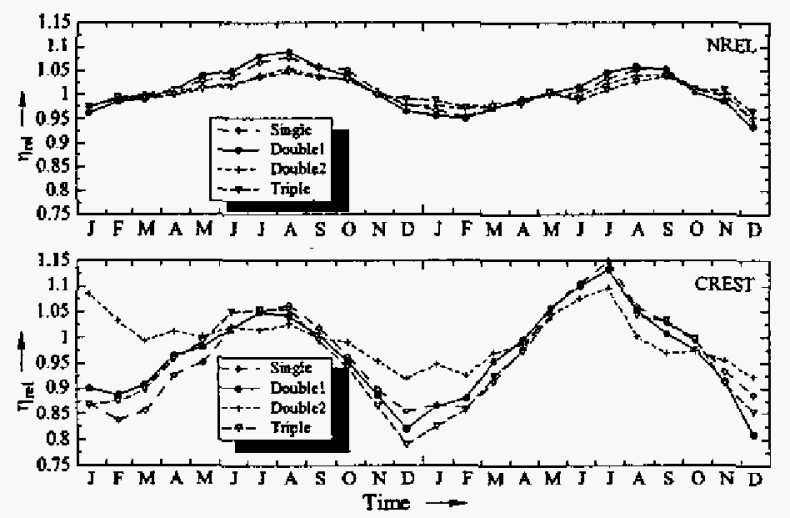

Fig. 1. Seasonal Variations of Monthly Efficiency.

\footnotetext{
${ }^{1}$ PR=annual-efficiency $\left.t_{\text {amm }}\right) /$ standard-efficiency $\left(\eta_{S T c}\right)$
}

spectrum (see e.g. [1]) or a temperature-induced annealing of Staebler-Wronski degradation (see e.g. [2]). These positive effects counteract the negative temperature coefficient of the open-circuit voltage ( $\left.V_{O C}\right)$. These effects are intertwined and a clear separation of their respective contributions is not easily achieved from outdoor data.

An approach is presented to separate these two distinct effects on performance and attempt to quantify their separate contributions for several devices operating at two different locations, with disparate operating conditions: the NREL test site in Golden, CO, USA and the test site of the Centre for Renewable Energy Systems Technology (CREST) in Loughborough, UK.

\section{EXPERIMENTAL ARRANGEMENT}

The devices under investigation have been operated for a minimum of two years at either test site. Care is taken to compare similar devices, although they are of different vintage and sometimes different manufacturers. Data presented here is for single junction, same band gap double junction (double1), dual band gap double junction (double2) and triple junction devices.

Both sites carry out full I-V scans, although there are some differences in their measurement approaches, which could affect the comparability, notably CREST having operated the modules at $V_{o c}$ between each 3 quadrant I-V scan, while the modules at NREL are operated at maximum power point in between 1-quadrant $\mathrm{I}-\mathrm{V}$ scans. Both double junction devices at CREST were installed 3 months prior to the beginning of measurements presented, so they were stitl in the initial stages of degradation during those first few months.

Data is summarized on a monthly basis: the monthly efficiency are calculated and normalized with respect to its calculated for the second year of study, when the devices are essentially stabilized. The analyzed data set was Iimited to cases where the irradiance was above $100 \mathrm{~W} / \mathrm{m}^{2}$, so that low light behavior would not dominate the analysis. Also, because there was a lot of activity at CREST during the summer of 2004, there may be some idiosyncrasies in the resulting aggregate values.

\section{SEASONAL EFFICIENCY VARIATION}

The aim of this paper is to investigate the seasonal variation in performance of amorphous silicon modules and identify the differences between the two sites. The two sites were shown to have very disparate climactic 
and operating environments [3], with differences both in the magnitude and spectral composition of the incident irradiance, and as well in operating temperature. The effects this has on average monthly efficiency data for modules situated at the two locations, is shown in Fig. 1.

The monthly efficiency exhibits smaller seasonal variations in Golden than it does in Loughborough. While the summertime peaks in performance attain similar order of magnitude values at both sites, but the winter declines are much more pronounced in Loughborough. It is also apparent that the inter-module variations are more pronounced at CREST. Although the cause could be due to the different thermal-operating regimes, this would be at variance with the findings of Rüther et al. [4], that locations with a warmer winter tend to experience less degradation. Hence, it is more likely to be due to different spectra or itradiance levels. In Loughborough, about $30 \%$ of the energy is generated at low-irradiance conditions, which proportionally weighs this operating condition more This will be investigated when looking at the seasonal shunt resistances, as done later in the paper. A further difference is the spectrum, which was discussed in some detail in [3], and is not duplicated here. However, it helps explain the differences in winter reductions, because Loughborough is significantly further north, and thus the spectral content of the direct-beam illumination tends to be more red-shifted than in Golden. The hypothesis that different mechanisms dominate the performance at the two sites is also supported by the fact that the minimum operating performance occur in different months: at NREL it appears in February, but while at CREST, it occurs in December). The maximum on the other hand (allowing for the idiosyncrasy in the second year of CREST measurements) is in both cases attained in August.

There would also seem to be different underlying mechanisms, because different cell types react differently. Double2 (dual band gap) modules overall show the least amount of seasonal variation in operating efficiency at both locations. Both single junctions, on the other hand, show larger seasonal variations. The triple junction has the most significant seasonal variations in Loughborough, while the Double1 module achieves the largest variation in Golden.

\section{FACTORS AFFECTING SEASONAL PERFORMANCE}

The hypothesis that the underlying causes are significantly different at both sites needs to be more supported and thus the seasonal efficiency variation is investigate more fully, using a simple delineation:

$$
\eta=\frac{I_{S C}}{G A} V_{O C} F F
$$

Where $I_{S c}$ is the short circuit current, $V_{\infty}$ is the open circuit voltage, $F F$ is the fill factor, $G$ is the incident irradiance and $A$ is the area of the module. A first order approximation is that Isc is linear with irradiance, thus pointing to three factors to investigate and identify as the possible mechanisms responsible for the seasonal behavior.
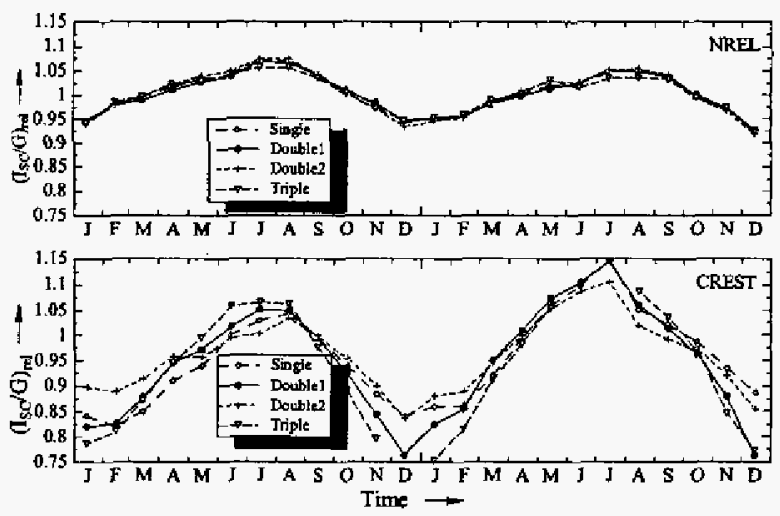

Fig. 2. Average Monthly $I_{s d} / G$

It is assumed that $I_{s c}$ is the main factor influenced by the spectral variations, and that once this is corrected for spectral fluctuations it does not subsequently pose any further effect on the devices.

The effects of variations in spectrum can be summarized by dividing the integrated short circuit current by the integrated irradiance in a given month. This results in significant seasonal variations of the photo-response, as illustrated in Fig. 2.

If the variations depicted in Fig. 2 are not due to thermal-annealing-induced absorption changes, then the devices at NREL have half their summer gain, and more than their entire winter loss, accounted for by spectral mechanisms. There is strong evidence that the spectrum is indeed the driver here (abeit some thermal annealing undoubtedly occurs), as give in [3]. The situation is different for the CREST devices, in that the losses in winter are more pronounced, and virtually all the summer gain is attributable to spectral effects. The differences amongst the devices are interesting: the dual band gap and single-junction modules experience the lowest variation, while the triple-junction device is the most affected. This is consistent with expectations because a triple junction needs to match the currents for the three junctions and thus the potential for spectral mismatch is greater.

However, this can explain at best only half the summer gain for the NREL site. Thus another positive influence must stem from either the changes in FF or $V_{0 c}$, where it is expected that thermally-induced changes in

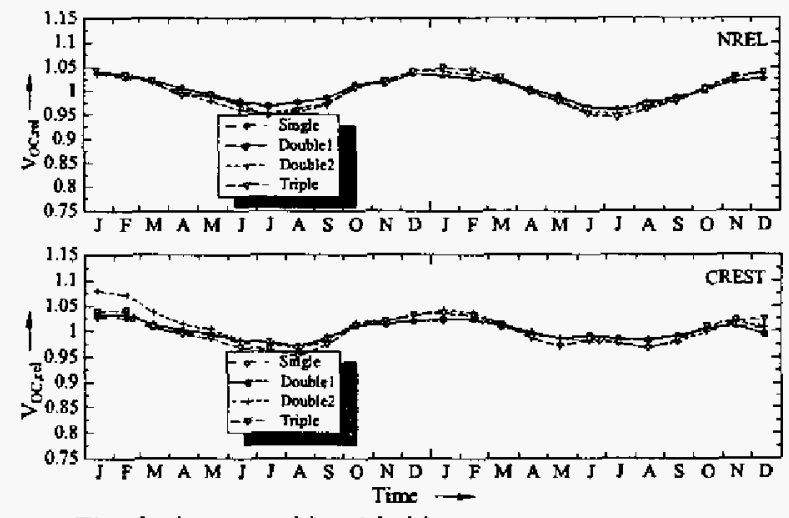

Fig. 3. Average Monthly $V_{\infty}$ 
carrier recombination will impact carrier life-time and mobility in a positive manner. First, the average $V_{O C}$ is considered in Fig. 3.

It is clear from Fig. 3, that $V_{O c}$ is not the driving factor behind the remaining summer increase. On the contrary. a further loss of about $5 \%$ is added, consistent with higher operating temperatures and the negative temperature coefficient of $V_{O C}$. This, however, does explain why the spectral losses can be larger than the seasonal efficiency variations, as a gain of $5 \%$ is achieved in winter, which offsets some of the spectral losses in winter.

The trends in $V_{\infty c}$ data between the two sites are surprisingly similar, although the summer reduction in Loughborough is slightly less pronounced than in Golden, which is attributable to the differences in temperature and irradiance between the sites. It is interesting to note, however, that the spread between the devices is minimal, even for Loughborough, where all previous measurements graphs in this paper exhibited a significant spread between the technology categories. The triple junction shows the largest seasonal variations in $V_{O C}$ at both locations, which will be due to the thermal properties of the multi-band gap material - the imadiance response is not so much different from the other materials. There are seasonal variations in the irradiance, which obviously would affect the open-circuit voltages, but this is not corroborated by the data in Fig. 3 , because the values portrayed represent averages that are convoluted by the opposing influences of irradiance levels and thermal effects. Here the number of measurements is important, and because of the longer days in summer, there are significant numbers of low-light measurements, especially in Loughborough, where many measurements are taken when the sun is behind the plane-of-array of the modules.

The missing $5 \%$ summer improvement for NREL performance data must then be attributable to increases in fill factor data. It appears that the performance variation for devices operated in Loughborough is largely explained by $I_{S c}$ and $V_{O C}$ alone. This seasonal variations in FF are illustrated in Fig. 4.

As expected from the previous discussion, there is a significant difference in the variations of the FF between the two sites. It is clear that there are differences in the mechanisms driving the seasonal performance fluctuations in both places.

In Golden, all devices follow a clear pattern, with a

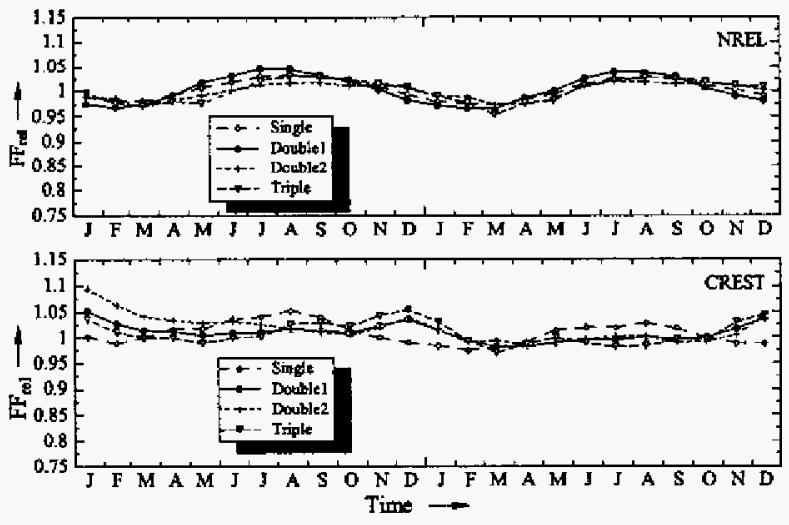

Fig. 4. Average Monthly FF seasonal variation of $\pm 5 \%$, achieving maximum values for most devices in summer. This clearly demonstrates the remaining contribution to the performance peak in summer and the nadir in winter. This variation cannot as shown below - be attributed to any other reason than seasonal thermal-annealing and thus a clear indicator of the importance of this mechanism in Golden.

Loughborough on the other hand, does not show such a clear pattem. The FF for the single-junction modules follow the same trend as the devices in Golden, attaining their maximum in summer. However, the multijunction devices behave very differently: they all show maximum values in winter. Although in the first year that could be attributed to the initial degradation, this cannot be the explanation overall, because it repeats in the second and third winter in this data set. This also counteracts some of the spectral losses in winter in Loughborough, as these were larger than the seasonal change in efficiency. The seasonal increase in FF data in winter appears surprising and might mean that red spectra cause a higher fill factor than blue spectra, which is contrary to what was published for single junction devices [5]. However, in the case of multi-junctions installed at CREST, the FF variations can be entirely attributed to current matching of the sub-cells, which was shown by a detailed model calculation of mismatch between cell and junction currents.

We conclude from this, that there is very little seasonal annealing observable in Loughborough, especially for multi-junction devices. There is a small amount of seasonal annealing observable for singlejunction devices. This could mean that there is very little recovery of the electronic properties in Loughborough and that it is of virtually no importance to the multi-junctions (which are less susceptible to degradation because of the larger built-in field). This could be due to the very mild summers in Loughborough, where there are rarely ambient temperatures above $25^{\circ} \mathrm{C}$.

There is also a type-specific difference in the modules operated at NREL: the temporal pattern of the same band gap double-junction module data precedes that of all the other modules by roughly two months. In both locations one finds that the modules with the lowest variations are the dual band gap ones.

The fact that the FF variations achieve their maximum in summer could also be attributable to the influence of irradiance levels. This can, however, be excluded because it is clear from Fig. 5 that virtually all devices attain maximum of FF value at mid-range irradiance. The data shown in Fig. 5 were segregated into irradiance bins of $50 \mathrm{~W} / \mathrm{m}^{2}$ size, indiscriminately for all two years under investigation and the bin average was calculated. Each device exhibits a distinct pattern, which could be due to the parasitic resistances as well as recombination, and current-matching between junctions in multi-junction devices. The normalization to STC values carried out in Fig. 5, leads to larger fill factors for most of the range. Larger fill factors are not a measure of operational quality - this has to be done in a much more detailed analysis. This behaviour is just used illustrate why the FF of some devices probably do not exhibit as large an increase as they could during summer months. 

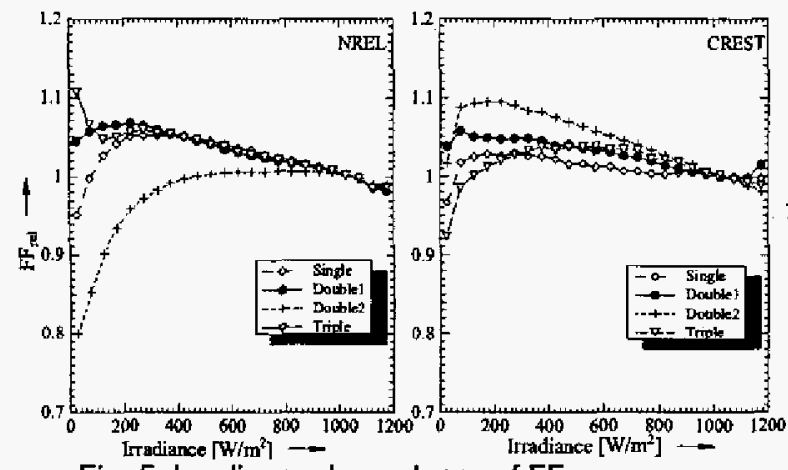

Fig. 5. Irradiance dependence of FF

The devices, which have the largest FF-variation are also the devices that exhibit the most significant dependence on irradiance. The device with the best stability in Loughborough (Double1) is also the one with the highest energy production, due to the disproportionate contribution from this irradiance range.

The seasonal behavior of the FF cannot, however, be explained by Fig. 5 and other reasons must be investigated. It is expected that there will be a negative temperature coefficient, because high operating temperatures also cause higher recombination, and so this can also be excluded. One possible reason for seasonal changes in the FF could be variations in the parasitic resistances, which should also be affected by seasonal annealing. In the following, these are investigated for NREL data only, as here more of an effect of seasonal annealing is seen, and the aim is to see if a variation in the life-time and mobility of charge carriers can be discerned in these parasitic resistances. The data shown in Fig. 6 are the stopes (dV/dI) of the I-V traces at open circuit $\left(R_{o c}\right)$ and at short circuit $\left(R_{s c}\right)$. Both parameters are superimposed of the parametric shunt and series resistance and contributions from the voltagedependent photo-current, which in turn is strongly influenced by the semiconductor quality. The data portrayed were limited to irradiances between 950-1050 $\mathrm{W} / \mathrm{m}^{2}$, which should remove any irradiance dependence of the parameters. The temperature range was not limited, because there is no temperature range that leaves a significant number of points in all seasons, thus
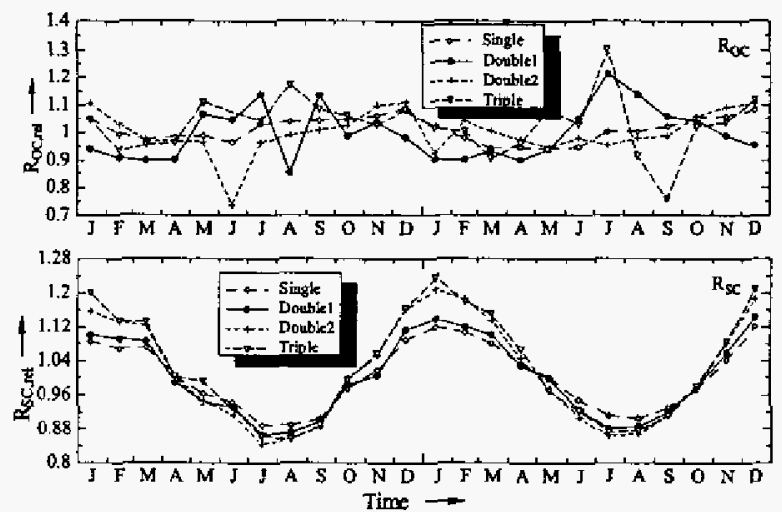

Fig. 6. Seasonal $\mathbf{R}_{\mathrm{SC}}$ and $\mathrm{R}_{\mathrm{oc}}$. there is about a $20^{\circ} \mathrm{C}$ seasonal variation in module temperature.

No apparent seasonal trend can be deduced from the variation of the Roc, but there is a very strong trend for $\mathrm{R}_{\text {sc. }}$. In the past it was shown that a strong functional relationship exists between irradiance and $R_{s c}$, but that should be excluded here due to the limitation of input values. The temperature does not have such a strong effect on the slopes and thus one could say that there is a clear seasonal variation in the $R_{s c}$, which could be explained by annealing.

\section{CONCLUSIONS}

The analysis of long term data collected at two sites has shown that significant seasonal variations in the operating efficiency of amorphous silicon devices exist. While the amplitudes of the variations are not too dissimilar, the mechanism driving the seasonal patterns were shown to be very different at both locations. At one site, Golden, US, the pattern is driven roughly in equal shares by seasonal annealing effects and spectral changes, while at the other site, Loughborough, UK, it is virtually dominated by spectral effects. This leaves the conclusion that there is no broad-brush solution to modeling the performance variations in these devices, nor is it possible to give a universally-applicable formula to quantify the magnitude of each of these effects - one has to look in each and every case into the drivers and compare with other sites.

\section{ACKNOWLEDGEMENTS}

R. Gottschalg is funded through an EPSRC Advanced Research Fellowship (EPSRC GR/T03307/01). J. del Cueto and NREL are funded by U.S. Dept. of Energy contract no. DE-AC36-99G010337

\section{REFERENCES}

[1] R. Gottschalg, et al., "Experimental Study of Variations of the Solar Spectrum of Relevance to Thin Film Solar Cells", Solar Energy Materials and Solar Cells, 79. 2003, pp. 527-537.

[2] J. A. del Cueto and B. von Roedern, "TemperatureInduced Changes in the Performance of Amorphous Silicon Multi-Junction Modules in Controlled Light Soaking", Progress in Photovoltaics, 7, 1999, pp. 101112.

[3] R. Gottschalg, et al., "Investigating the Seasonal Performance of A-Si Single- and Multi-junction Modules", Third WCPEC, Osaka, 2003

[4] R. Rüther, et al., "Performance Test of Amorphous Silicon Modules in Different Climates - Year Two: Higher Minimum Operating Temperatures Lead to Higher Performance Levels", 20th EUPVSEC, 2004, pp. 16171620.

[5] R. Rüther, et al., "Spectral Effects on Amorphous Silicon Solar Module Fill Factors", Solar Energy Materials and Solar Cells, 71, 2002, pp. 375-385. 